\section{Cahiers de Narratologie}

Analyse et théorie narratives

13 | 2006

Nouvelles approches de l'intertextualité

\title{
L'absolu intertextuel dans l'exégèse de Paul Claudel
}

\section{Didier Alexandre}

\section{CpenEdition}

\section{Journals}

Édition électronique

URL : http://journals.openedition.org/narratologie/308

DOI : 10.4000/narratologie.308

ISSN : 1765-307X

Éditeur

LIRCES

\section{Référence électronique}

Didier Alexandre, «L'absolu intertextuel dans l'exégèse de Paul Claudel », Cahiers de Narratologie [En ligne], 13 | 2006, mis en ligne le 01 septembre 2006, consulté le 14 novembre 2019. URL : http:// journals.openedition.org/narratologie/308 ; DOI : 10.4000/narratologie.308

Ce document a été généré automatiquement le 14 novembre 2019.

\section{(c) $(1) \&$}

Cahiers de Narratologie - Analyse et théorie narratives est mis à disposition selon les termes de la licence Creative Commons Attribution - Pas d'Utilisation Commerciale - Pas de Modification 4.0 International. 


\title{
L'absolu intertextuel dans l'exégèse de Paul Claudel
}

\author{
Didier Alexandre
}

Le moment est venu pour moi aussi que cette vision s'empare de moi qui est d'être vu pour voir, et cette parole pour parler qui est d'être parlé !1

1 Dans ses exégèses, Claudel raconte volontiers dans quelles circonstances il fut amené à commenter les textes bibliques. « Un imprimeur, nommé Pichon, » lui demanda en 1927 « d'écrire une préface à une nouvelle édition qu'il préparait de l'Apocalypse ». Le poète refusa : mais la «proposition, nuit et jour, ne [le] laissait pas en repos. » Ce qui aurait dû être "l'affaire de quelques jours, de quelques semaines au plus » occupa pendant près de vingt-huit années celui qui, les Otage, Soulier de satin et Tête d'Or achevés « était vacant ».

J'avais soixante ans, ai-je dit, à ce moment, j'en ai maintenant plus de quatre-vingttrois, et il n'est pas vraisemblable qu'autre chose que la tombe vienne mettre fin à l'investigation téméraire et passionnée où je me suis trouvé engagé par un enchaînement progressif de questions et d'attraits qu'il n'était pas en mon pouvoir d'éluder. Seigneur, j'ai aimé la beauté de Ta maison, dit le Psaume. Quelle maison comparable à l'Écriture qui est le temple de la pensée divine ${ }^{2}$

2 En fait, on ne saurait limiter cette fréquentation de l'Ecriture à cette durée. Claudel est un catholique pratiquant, qui connaît parfaitement son missel et son bréviaire, qui a lu de première ou de seconde main les théologiens (Thomas d'Aquin, Augustin), qui est informé des questions liturgiques... La «maison" catholique constitue un immense champ intertextuel, constamment et immédiatement présent à la mémoire et à la conscience du poète lorsqu'il écrit, que le poème soit une création théâtrale ou poétique ou essayiste. L'écriture claudélienne est constamment intertextuelle.

3 La première finalité de l'intertexte biblique est commentative. La démarche claudélienne face à un objet, qu'il soit objet réel ou texte, nous reviendrons sur ce point, est interrogative : le poète exégète a répété, dans ses entretiens avec Frédéric 
Lefèvre, les Mémoires improvisés, ou dans son Mallarmé, la catastrophe d'Igitur, sa fidélité à l'enseignement de celui dont il fréquentait les mardis Rue de Rome et qui «le premier [s'est] placé devant l'extérieur (...) comme devant un texte, avec cette question : Qu'estce que ça veut dire? $»^{3}$ L'intertexte inter - vient comme l'élément qui permet d'établir la réponse. Un sens, et une intentionnalité de sens, sont posés comme préalables à toute interrogation : un texte, quel qu'il soit, est énigmatique - mais d'une énigmaticité que l'intertexte est appelé à lever. L'écriture naît d'une interrogation - Paul Claudel interroge l'Apocalypse; Paul Claudel interroge le Cantique des Cantiques -, où le préfixe inter - du verbe interroger retrouve sa valeur relationnelle et interactive. Et de fait, Claudel ouvre bien des poèmes, par exemple Villes ou Le Contemplateur de Connaissance de l'Est, par une question ou un paradoxe, comme il commence souvent une exégèse par l'affirmation d'un désordre et d'une incohérence : il écrit du Livre de Job :

Dans les reprises acharnées de cet épouvantable sanglot, tout est désordre et contradiction ${ }^{4}$.

4 Mais ce désordre et cette incohérence se situent moins dans le texte à commenter que dans les insuffisances du lecteur. La pratique intertextuelle implique une lecture attentive aux échos, qui prenne le texte dans sa successivité et sa simultanéité - une lecture qui passe non pas par la vue, mais par l'écoute : il faut que l'œil écoute. Pour rendre lisible par l'intertexte le texte visible, il faut donc créer le lecteur propre à cette lecture. L'intertexte lève l'énigme du texte et l'énigme du moi - il interroge autant le commentaire que celui qui le fait :

[Dieu] a besoin de notre bonne volonté active pour que cette réalité qu'il nous révèle devienne en nous une image, non pas morte, mais, comme Lui, vivante et énergique. Nunc videmus per speculum in aenigmate, nous dit la première Corinthienne $(13,12)$. En regardant, en écoutant de toutes nos forces, patiemment et passionnément, la parole de Dieu, nous enseignons, peu à peu, à voir, à vivre et à parler, à cet enfant de Dieu aveugle, enveloppé et paralysé, qui dort au fond de chacun de nous. Si nous sommes assez simples, assez purs et assez patients, nous appréhenderons, à travers la surface accidentelle des objets qui nous sont présentés, l'essence. Nous comprendrons ce qu'ils veulent dire ${ }^{5}$.

5 Cette mutualité informante, du sens et du sujet, qui n'est pas étrangère à la conaissance réalisée à travers la connaissance, passe par la Parole et donc l'intertexte. Ainsi, pour dire sa pratique intertextuelle dans la citation de J'aime La Bible faite précédemment, Claudel recourt explicitement à la citation du Psaume 25 (verset 8), comme si l'intertexte lui renvoyait l'image de sa propre pratique. C'est cette mise en ordre du texte et du moi dans et par la parole de Dieu - l'intertexte - que je souhaite examiner dans les pages qui suivent.

Le matériau intertextuel

6 Le matériau intertextuel claudélien couvre des domaines très diversifiés : littéraire (par exemple la parfaite connaissance de Rimbaud, de Mallarmé, des traductions de Leconte de Lisle), philosophique (par exemple, la connaissance de Thomas d'Aquin, Kant, Taine, les théories matérialistes fin de siècle), scientifique (par exemple, la connaissance de Claude Bernard ou des ouvrages du docteur Alexis Carrel), biblique, religieux. Nous nous limiterons à ce dernier domaine, sans nous en tenir uniquement à l'intertexte explicite.

7 Ce champ biblique, - comme tout champ intertextuel-, on peut l'aborder en compréhension et en extension. Le trait commun qui fait l'unité du texte biblique 
claudélien se situe dans son énonciation et dans l'événement central qui en donne le sens. Claudel, fidèle à la leçon des Pères de l'Eglise, unifie la Bible en lui reconnaissant, évidemment contre l'exégèse historiciste développée depuis Spinoza et le protestantisme, un locuteur unique. Il passe outre ainsi les différences génériques, tonales, thématiques (livres historiques, livres prophétiques, poèmes, livres sapientaux etc.), en les renvoyant à un seul auteur.

c'est que précisément la Bible n'est pas un amas de documents hétéroclites, c'est un édifice composé de matériaux intelligents, ou plutôt c'est un être vivant que nous voyons croître et se développer sous nos yeux, comme le gland qui sait dès le commencement qu'il sera chêne, et qu'il ne peut pas devenir autre chose inexorablement qu'un chêne. Partout, à travers des truchements divers, des langages divers, des formes diverses, des occasions diverses, nous retrouvons le même auteur qui a à nous parler de la même chose, conscient du même trésor et usager du même répertoire.

Cet auteur, nous autres, fidèles catholiques, nous le savons, c'est l'Esprit Saint ${ }^{6}$.

8 Sans nier la personne des prophètes, auxquels il rend leur statut de personne parlant à la place de Dieu, il soumet donc le texte à la plus forte des figures auctoriales, Dieu, et donc à une omniscience absolue qui cautionne, en dehors de toute temporalité humaine d'écriture et de lecture (rédactionnelle, ordre linéaire des livres qui se succèdent), les déplacements à l'intérieur du champ. Le texte porte la marque de l'éternité divine, et il est donc simultané dans sa conception et dans la vision que l'exégète peut en avoir. Le concept d'intention, que Claudel emprunte à la pensée thomiste, en le déplaçant du domaine de la morale de l'action au domaine du sens, fonde par un finalisme indissociable d'un esprit ordonnateur les relations de «correspondances » ou d'« allusions » que l'exégète pourra établir. L'intention est en effet le mouvement d'une volonté qui se meut elle-même vers un objet et qui meut toutes les autres puissances de l'âme vers cet objet $^{7}$ : déplacée sur l'écriture, ceci implique que tout élément textuel est porteur de virtualités voulues par l'auteur. Tout texte à commenter est donc chargé d'un sens potentiel et intentionné :

Si la Bible est vraiment la Parole de Dieu, avec quel respect total, avec quelle attention fervente, avec quelle ingéniosité à lui apporter tous les renforts et résonances favorables devons-nous en étudier les intonations, les démarches, les procédés de composition et de développement, et par-dessus tout dans le multiple jeu des allusions et des correspondances, l'intention! (...) Nous n'avons plus devant nous un bric-à-brac de bazar, une multitude de petits objets incohérents qu'il s'agit de réduire en poudre par un travail obstiné, mais d'immenses étendues synclinales, un jeu de couches diverses, paraissant, disparaissant, et bouleversé par des émersions, un énorme banc de témoignages que l'on ne peut comparer qu'aux craquements et aux reprises de la géologie, une signification à perte de vue ! C'est Dieu même qui, d'une main froisse, pétrit, compose l'étoffe et le dépliement de Sa Création, et, de l'autre, Se donne la peine de nous l'expliquer, à sa manière, qui est différente de la nôtre ${ }^{8}$.

9 Les métaphores naturaliste (le gland qui contient virtuellement, en puissance, le chêne) et géologique inscrivent la totalité dans ces métatextes : elles sont indissociables de la position d'un observateur (Claudel écrit que le gland sait qu'il sera chêne - en fait, le gland ne sait rien, mais Claudel le sait), dont le point de vue totalisateur se confond avec celui de Dieu. 

l'« ingéniosité » et des « renforts », et non pas seulement de "l'attention fervente ", réponse dans la foi à l'intention. Cette inventio, qui se traduit dans le commentaire par le recours à l'imagination inventive, voire débridée, de Claudel, porte sur la médiation symbolique qui doit être établie entre le texte à commenter et le sens intentionné. C'est pourquoi, dans $\mathrm{Du}$ sens figuré de l'Ecriture, ou dans Les Aventures de Sophie, ouvrage contemporain du traité, Claudel privilégie l'esprit sur la lettre : l'intertexte ne s'impose à partir d'un texte premier que parce qu'il médiatise le sens à inventer - c'est-à-dire à trouver ou retrouver.

Or, ce que le corps est à l'âme, la lettre l'est à l'esprit. Les mots et les phrases ne valent que par le sens, par la vérité dont elles sont prégnantes. En perdant l'esprit, elles cessent de respirer, elles ne sont plus que des espèces inertes et matérielles, quelque chose qui non seulement est la mort, mais qui la procure. Hélas! nous ne voyons que trop cette funeste transmutation opérée sur la parole de Dieu aux mains des exégètes littéralistes ${ }^{9}$.

11 La notion d'esprit permet à Claudel de ne pas fixer le texte et donc l'intertexte biblique dans une signification unique. La question de la traduction du texte hébraïque est examinée dans le seul but de justifier la polysémie et donc la multiplicité des interprétations, même les plus surprenantes. Le texte biblique contient en lui l'inventio. A un sens fidèle à la philologie, à la " grammaire " et au "dictionnaire ", «tous deux déficients ", Claudel préfère un sens établi par une compréhension interne de la Bible, fondé sur la memoria. Prenant appui sur l'ouvrage de Paul Vuillaud, La Clef traditionnelle du judaïsme, Claudel revendique le droit de préférer à la citation textuelle la référence indicielle, l'essentiel étant le « soutien d'autorité »:

"Les anciens théologiens du judaïsme", dit encore M. P. Vuillaud dans le même ouvrage, (...) "n'avaient pas l'usage de toujours citer textuellement. Ils citaient l'Ecriture asmak ta b'alma (comme soutien d'autorité), et cet appui a posteriori était trouvé dans le texte sacré de toutes manières, c'est-à-dire que souvent le plus faible indice suffisait pour fortifier la trame dialectique." (Cela fait penser à notre sens accommodatice) "Il leur arrivait de plier la lettre afin de l'accommoder à la signification d'un contexte"10.

12 Claudel se méfie donc des traductions qui fixent le sens, en vertu d'un attachement à la polysémie de l'hébreu où il investit la marque de l'Esprit et sa propre pratique des échos et consonances textuelles.

L'intention qui guide l'écriture divine est un point fixe autour duquel s'ordonnent l'Ancien et le Nouveau Testament. Il s'agit, bien évidemment, du sacrifice christique. De cette démarche qui pense un texte en fonction de cet événement central qui donne le sens à partir d'un intertexte, voici un exemple emprunté au Livre de Tobie :

Cependant le chrétien (...) a plusieurs fois tressailli ! Après tout il s'agit d'une rédemption ! Cette Sara, là-bas, au fond de l'Asie, opprimée par le diable, mais c'est la nature humaine, l'âme humaine, reflet de la sagesse éternelle, à qui l'Ecriture partout donne figure de femme. Tobie, fils de Tobie, c'est le Fils issu et envoyé de Son Père. Le poisson nous est familier : c'est l'image au fond des catacombes que nous trouvons sous la corbeille des pains eucharistiques ${ }^{11}$.

La démarche de Claudel suit cet ordre : Sara = âme humaine, sagesse, la médiation étant donnée par l'Ecriture (intertexte) ; Tobie, fils de Tobie = le Fils du Père, la médiation étant fournie par le poisson capturé par Tobie dans le fleuve Tigre interprété par 
Claudel conformément à la symbolique chrétienne. L'identification se fonde sur un trait commun, la féminité, ou sur des rapports analogiques (la filiation, le poisson est à Tobie ce que le poisson est au Christ). Ce fragment illustre ce principe énoncé par Claudel dans Du Sens figuré de l'Ecriture :

L'Ancien Testament est incompréhensible sans le Nouveau, qui vient tout remplir, justifier et expliquer. Je ne suis pas venu, dit Notre-Seigneur, dissoudre, mais remplir. Pas un iota de la loi ne passera et pas un point sur l'i (Matth., 5, 18). Et, comme en réalité toute la lettre a passé, il faut bien admettre, si quelque chose subsiste, que ce quelque chose est le sens spirituel subjuguant et inhérent jusqu'aux moindres détails du texte premier $^{12}$.

Aussi le statut du Christ claudélien est-il spirituel, c'est-à-dire textuel. La venue du Christ est pensée en termes de textualité, sur le mode hyperbolique : " A chaque instant reviennent dans l'Evangile ces mots: ut adimpleantur Scriptura.» Ou la «transfiguration » est « la clarification, l'illumination d'un texte opaque. " ${ }^{13}$ Claudel avoue ainsi, implicitement, que l'événement christique est l'intertexte central qui donne la transparence au reste du texte biblique. Dans Un poète regarde la Croix, ou dans L'Epée et le miroir, de peu postérieur, on trouverait aisément des pages où s'accomplit cette textualisation du Christ en croix, une croix dont la géométrie dessine un centre où les quatre directions, la pluralité des espaces - et des textes -, viennent se croiser pour établir un point fixe et central, ordonnateur, signifiant :

Voici la page tournée qui éclaire tout, comme cette grande feuille illustrée sur le missel que connaissent les prêtres quand ils se préparent à lire le canon, car n'est-il pas dit qu'à la tête de tout le Livre il est écrit de Moi? (Ps. XXXIX, 8) La voici, éclatante et peinte en rouge, la grande page qui sépare les deux Testaments!

(...)

il est écrit : Quand je serai élevé je tirerai tout à moi. Tout arrive à notre rencontre. Tout vient se mesurer à ce double diamètre d'un cercle invisible, qui discerne le droit et le gauche et le haut et le bas. Tout ce qui passe vient demander l'heure à ce gnomon surméridien. Tout ce qui est capable de signification vient lui demander ses raisons d'être ${ }^{14}$.

16 L'extension du champ biblique intertextuel claudélien est donc considérable. Dans $D u$ sens figuré de l'Ecriture, Claudel définit un premier ensemble composé des deux testaments, dont il ne sépare pas les interprétations qui ont pu en être données par les Pères de l'Eglise, par le dogme catholique et donc la théologie, et dans la liturgie catholique.

Ce champ comprend plusieurs zones de clarté : il y a les figures que Notre-Seigneur a pris la peine de nous expliquer Lui-même dans ses paraboles; il y a les interprétations des apôtres et des Pères : il y a celles que l'Eglise a faites siennes en les incorporant dans sa doctrine et dans sa liturgie ${ }^{15}$.

17 Claudel construit ainsi un ensemble intertextuel par métonymie, tout commentaire ou tout symbole intégré par l'Eglise ayant valeur d'autorité, c'est-à-dire étant marqué par la spiritualité de l'Esprit saint. A ce mouvement d'intégration n'échappe ni le réel ni la littérature même.

18 Lorsque, dans la dernière citation faite du Livre de Tobie, Claudel rappelle que «l'Ecriture partout donne figure de la femme » à la «sagesse éternelle »; il ajoute par une note: 
Se rappeler la parabole de Rimbaud: L'Epoux infernal ${ }^{16}$. référent d'un terme qui apparait dans le texte biblique - que ce référent soit l'objet désigné ou un objet associé par métonymie - est ainsi inscrit dans une structure communicationnelle, qui va de l'énonciation par Dieu à la compréhension humaine, en passant par la constitution de la phrase grâce au lexique et à la grammaire : c'est pourquoi pour Claudel «tout est symbole dans la nature et tout est parabole dans l'événement ${ }^{18}$. Dans les ouvrages où Claudel réfléchit sur le texte biblique, la métaphore linguistique est fréquente.

Le Verbe, pour communiquer avec nous, pour nous écrire cette longue lettre qu'est Son enseignement, pour que nous nous entendions avec Lui, a eu besoin d'un langage et premièrement d'un vocabulaire. Ce vocabulaire n'est point fait de main d'homme, d'expressions fabriquées. Il n'est autre que la Création elle-même, cet inépuisable répertoire d'images significatives du Créateur qu'est la Création. (...) Tout le vocabulaire de l'Ecriture n'est fait que de termes concrets, que de mots tout prêts, dans l'appel qu'ils font à d'autres mots, à apprendre ce qu'ils veulent dire. Images de Dieu qui ne demandent qu'à consommer à ses pieds un sacrifice de significations.

Le monde n'est pas seulement un vocabulaire. C'est à lui que nous empruntons tous les éléments de notre grammaire. (...) La nature a trouvé le moyen de se faire entendre non plus seulement à notre oreille, mais à notre intelligence. Elle parle.

Elle ne parle pas toute seule comme une folle. Elle parle de quelqu'un et elle parle à quelqu'un ${ }^{19}$.

21 Même s'il se réfère à un modèle oral - Dieu parle -, Claudel pense le réel sur le modèle de l'écriture - et ce, dès son Art poétique rédigé de 1900 à 1904. Dieu nous écrit « cette longue lettre", d'un monde qui s'ordonne comme une phrase. L'écriture qui est première fait de tout objet un gramme au sens où l'entend Derrida: la préséance accordée à l'esprit sur la lettre transforme en gramme généralisé le réel, en inscrivant en lui le sens, que Claudel identifie à la différence essentielle ou la différence ontologique, et en creusant la différence entre l'être là du monde et son vouloir dire.

L'entretien : digression ou unité ?

22 Le commentaire claudélien suit une démarche associative, que les termes d'allusion, d'écho, d'association, de référence ou de latéralité, et la place faite à la figure, comparaison, métaphore, analogie, peuvent bien résumer ${ }^{20}$. L'établissement du sens passe par le sens de la liaison, des liens, ou encore des «connexions » ${ }^{21}$. Le Christ luimême n'échappe pas à cette fonction de lien définie dans le contexte textuel : il est «point de suture »- et on sait, depuis $S / Z$ de Roland Barthes ce que le texte doit au 
tissage -, ou encore, en terme d'écriture musicale, « intervalle ». La métaphorisation de la démarche met, elle aussi, l'accent sur ce qui est entre et qui remplit une fonction de liaison et de transformation, tel le feu qui « s'allume et s'entretient à tous les matériaux et réunit tout ce à quoi il se prend en une seule langue de flamme claire » et dont le modèle est donné par (l'intertexte de l'épisode évangélique de) la Pentecôte ${ }^{22}$. Il faut ainsi donner au préfixe entre sa pleine valeur : placer entre l'intertexte, c'est combler un vide, mais c'est faire s'entretenir, dialoguer ensemble, apporter l'un à l'autre sa différence, deux éléments textuels différents. A maintes reprises, Claudel fait de l'intelligence la faculté qui interprète le texte. L'intelligence demeure, pour Claudel, la faculté intervallaire qui suppose une pénétration des objets textuels pour en saisir le principe et pour, dans un second temps, les réunir. Elle est donc analytique, procédant par décomposition et recomposition: comprendre, c'est prendre ensemble des éléments disjoints pour les conjointer. Mais l'analyse ne porte pas sur le sens littéral: elle porte sur le sens spirituel, et implique donc que l'intelligence soit spirituelle, ou inspirée. Tel est bien le sens qu'il faut donner à la définition de l'intelligence donnée dans l'Art poétique :

"Inlelligere", inlire. "Lire", s'assimiler et le sens au sens. "Comprendre", saisir en même temps, réunir par la prise. Comme on dit que le feu prend, ou que le ciment prend, ou qu'un lac se prend en hiver, ou qu'une idée prend dans le public, c'est ainsi que les choses se comprennent et que nous les comprenons ${ }^{23}$.

La notion d'interprétation résume la complexité de la fonction de l'interprète. L'interprète est à la fois le traducteur de la parole de divine, celui qui joue cette parole, et celui qui, intermédiaire, se prête au jeu. Il est donc à la fois actif, par son intelligence analytique, et passif, par son abandon au jeu. Dans Du sens figuré de l'Ecriture, Claudel fait un long excursus sur "Les langues et leur interprétation». Celle-ci, définie en référence à des figures ou des prophètes bibliques, Zacharie, David ou Isaïe, ou à l'épisode de la Pentecôte, suppose l'inspiration, ou encore le "don des langues", la capacité à surmonter les «barrières » linguistiques, à parvenir, en passant outre les différences linguistiques des langues nationales, au sens ${ }^{24}$. Cette part d'irrationnel, ou encore d'anima complétant le raisonneur animus, fait de tout exégète un inspiré, dont l'intelligence est soumise à la foi et à l'amour :

Dieu est amour et la faculté en nous qui est propre à l'appréhender, ce n'est pas l'intelligence, elle ne fait que déblayer le chemin et établir les connexions, c'est l'amour ${ }^{25}$.

24 L'intertexte conserve ainsi une fonction auctoriale et autoritaire forte qui minimise la part prise par l'exégète. Cette humilité devient en fait la garantie spirituelle qui cautionne la pertinence du commentaire. La pratique intertextuelle établit le sens vrai, parce que le champ intertextuel a pour origine l'esprit saint, et parce que l'interprète est soumis à l'esprit saint :

d'une manière générale ou peut dire que l'inspiration, jouant souvent le rôle d'une intervention, ou même d'une adjonction, transcendante dans notre ligne de vie moyenne, tient dans notre activité psychologique une place essentielle et indispensable ${ }^{26}$.

La lecture par l'intertexte obéit donc à une progression de l'humain vers le spirituel, tant dans la signification que dans l'énonciation, d'une parcellisation, suivie de l'établissement de connexions, à une totalisation, l'ensemble du mouvement étant pensé, dans J'aime la Bible, sur le modèle de l'arbre de vie, dont « la bouture insérée aux 
premières pages du Livre sacré a fructifié aux dernières " pour s'accomplir dans la croix ${ }^{27}$. Que ce soit dans un commentaire ponctuel, ou dans la constitution globale d'un commentaire, Claudel procède de la partie au tout, du mot à la phrase, du fragment à la composition. Faute de place, je choisirai deux exemples, le premier emprunté au Livre d'Esther, le second à La Rose et le rosaire.

Dans les dernières pages du commentaire du Livre d'Esther, Claudel commente cette parole d'Assureus à Esther :

Accede tange sceptrum Esther. - Et comme elle se taisait, il prit la verge d'or et la posa sur son cou, et il l'embrassa et lui dit : Pourquoi ne me parles-tu pas ?28

Le commentaire procède de la partie au tout, du mot (sceptre) à la compréhension de l'intégralité de la scène, en trois étapes, de deux manières. Claudel part de l'objet concret, le sceptre, qu'il décompose, ou plutôt analyse par dissociation, selon une démarche abstractive, en fonction des facultés humaines, thomistes, la volonté et l'intelligence et la sensation, et des domaines impliqués, vouloir, pouvoir, agir, savoir.

Le sceptre, c'est le manche, le levier de commande, l'instrument de gouvernement et de domination. C'est l'insigne de la rectitude, la règle, la ligne de report et de mesure. Et c'est aussi l'extension au dehors de notre acte et de notre pensée, la rallonge de notre personne, la coudée que nous nous ajoutons, le trait d'union, l'index, l'antenne, le rayon, le moyen de connaissance, de communication et de puissance.

Le modèle mécanique (avion?, radio), le modèle mathématique côtoient la référence (soulignée par moi en gras) aux évangiles de Matthieu (VI, 27) ou de Luc (XII, 25) : «Qui de vous, en s'inquiétant, peut ajouter à son âge une seule coudée? » Claudel montre ainsi la vanité de ce plan humain par cet intertexte christique, et, implicitement, la nécessité du passage à un autre plan interprétatif ${ }^{29}$. Celui-ci est référé à la fois par la médiation de l'Ancien testament et du Nouveau testament:

C'est la verge qui accomplit tant de merveilles aux mains de Moïse et d'Aaron. C'est le sceptre qui ne sera pas ôté de Juda, pas plus que la croix aux chrétiens. C'est le sceptre même que nous avons entendu tout à l'heure Esther supplier son époux de ne pas remettre à ceux-là qui n'existent pas ${ }^{30}$. La dernière étape réconcilie le plan humain et le plan divin, l'inscription de la volonté humaine, et donc du savoir, du pouvoir, de l'agir, dans la volonté divine, exemplifiée par Moïse ou Aaron. Par la médiation intertextuelle, le sceptre est devenu croix, si bien qu'Esther est à ce stade associé au contexte christique :

Voici maintenant qu'il le lui met sur son cou à elle, signifiant la croix et ce joug qu'il est bon à nous de porter dès notre adolescence (Thr.) ${ }^{31}$

Cette conciliation de l'humain et du divin, du concret et de l'abstrait, du corps et de l'esprit s'achève dans la référence à la Vierge Marie. En fait, nous avons omis l'identification faite entre Esther et la Vierge dès l'introduction de la citation, reprise au terme du paragraphe :

Incipit :

Et ici se place cette parole solennelle, cette sainte formule d'un amour royal que nous aimons tant à répéter, le jour de l'Assomption de la Sainte Vierge : Accede [...]

Clausule :

Voici l'Humanité en la personne de la Sainte Vierge, admise à mettre le regard et la main au gouvernement de toutes choses. Il ne convenait point que le sceptre fît défaut à la couronne. 
lexte de l'Ancien testament prend sens par le Nouveau testament. Claudel, dans ses autres exégèses, reprend cette identification d'Esther à la Vierge Marie ${ }^{32}$. Cette intertextualité restreinte à l'œuvre se vérifie dans l'œuvre poétique, le poème Tange sceptrum, Esther (Po, p. 831), et dans le théâtre, puisque Claudel intègre cette lecture dans le programme de la Comédie française pour les représentations de L'Annonce faite à Marie de $1955^{33}$. Or, cette permanence ne se fonde pas sur la liturgie du jour de l'Assomption, et aucun passage du Missel ou du Bréviaire ne permet d'associer les deux figures. Peut-être l'intertexte de L'Année liturgique de Dom Guéranger, connu de Claudel, permet-il cette interprétation, ainsi que certaines antiennes de la fête de l'Assomption ${ }^{34}$.

Ce progrès de la partie au tout, dont chaque étape passe par des relais intertextuels, se vérifie dans la constitution des grands commentaires. Claudel part du mot, en conservant le projet de commentaires totalisateurs. Ainsi Emmaüs, puis Emmaüs II, appartiennent à un ensemble inachevé, un commentaire chronologique de tout l'Ancien testatment ordonné autour de la page centrale, christique. C'est de cet ensemble que sortent aussi les commentaires Jérémie, L'Evangile d'Isaïe, et l'Introduction à Isaïe dans le mot à mot. L'intertexte possède ainsi une vertu unifiante : la notion d' " unité » est au centre de ce dernier essai méthodologique, autant à l'échelle du texte d'Isaïe qu'à celle de la Bible tout entière. Ce fractionnement des publications, et l'échec relatif, puisque Claudel ne mène pas ce projet à terme, montrent les limites de la démarche totalisatrice qui viserait à enfermer l'histoire du monde dans les limites du livre fondé sur le Livre - projet mallarméen, s’il en est, où la Présence de Dieu, centrale, remplace le Néant. Ceci se vérifie de nouveau dans les commentaires construits autour de la figure de la Vierge, dont sortiront Paul Claudel interroge le Cantique des Cantiques, dont la forme varie du mot à mot, mené verset après verset, au dialogue et à la méditation, et La Rose et le rosaire, ensemble de textes hétérogènes réunis par le modèle récitatif du rosaire donné par la liturgie ${ }^{35}$.

que la totalisation et l'unification dont l'efficacité se vérifie à l'échelle du paragraphe ou de la page sont contrariées par la parcellisation même du mot à mot. La médiation intertextuelle, on s'en est rendu compte, est accumulation de références sur un mot prélevé dans le texte à commenter. Claudel valorise ainsi l'hypotexte par rapport à l'hypertexte : mais ce faisant, il soumet son texte au risque de la digression. Du reste, les « excursus ", " notes ", « digressions ", " disquisitions » ou " appendices » ne manquent pas dans les commentaires. Claudel avait envisagé de donner comme titre à ce qui est devenu Au milieu des vitraux de l'Apocalypse «Conversation Divagations Rêveries sur l'Apocalypse ».

L'exégèse d'un objet, ou d'un mot qui le désigne, peut ainsi apparaître au lecteur comme une digression ${ }^{36}$. Ainsi dans Un poète regarde la croix Claudel introduit brutalement, même si le calice a sa place dans le récit de la Passion christique, le couple «Calice-Lys ». Il associe au lys, puis au calice, un ensemble de références intertextuelles impressionnant, qui va de "l'imagerie symbolique de toutes les religions » aux textes bibliques, les uns agglomérés autour du lys (Psaume, 68, 3; Osée, 14, 6; Cantique des Cantiques, 2, 1 ; en note 3 Rois, 7, 26), les autres autour du calice (Jean, 8, 23 ; Colossiens, 3, 2 ; Matthieu, 26, 39 et Jean, 19, 30, Genèse, 2, 21, 9, 21, Psaumes, 77, 65, Matthieu, 20, 22, Psaumes, 80,11, 44, 11, 1 Corinthiens, 10,16, Psaumes, 72, 26, et neuf références placées en note à Isaïe, Habacuc, Jérémie, Ezechiel, Psaumes) ${ }^{37}$. Cette accumulation éloigne le lecteur et du sujet de l'exégèse, les paroles prononcées par le Christ sur la Croix, et du texte qui 
en est le support, présenté dans le cadre d'un office du vendredi saint entendu en 1933 dans une église $d u$ " Nouveau monde $»^{38}$. Cette rupture, constatée au niveau textuel - la lettre - n'en est plus une si le développement est réinscrit dans l'ensemble du sens spirituel. Un poète regarde la croix n'est pas soumis, comme L'Epée et le miroir, au travail de segmentation, de découpe par l'épée suivi d'une recollection médiatisée par le miroir révélateur du sens. Claudel situe le moment d'énonciation et de rédaction de son commentaire le jour du vendredi saint: il écoute les quatre évangiles et recueille la diversité des témoignages sur la Passion du Christ. Le symbole est la coupe, «le vase amer qui permet de tout comprendre", définie par la médiation de l'épisode de la coupe dans laquelle Joseph boit et avec laquelle il pratique la divination ${ }^{39}$. Dans le commentaire, Claudel se compare à Benjamin, le plus jeune des frères de Joseph, qui découvre dans son sac la coupe de Joseph. Il se définit ainsi par analogie : Joseph est à Benjamin ce que le Christ est à Claudel, et la coupe devient ainsi le calice amer que l'on donne à boire à tout chrétien le vendredi saint. Ainsi, le développement lys-calice met l'accent sur l'événement central, le lys-Christ, qui ordonne l'exégèse, et sur l'acte même du commentaire, le recueil dans le « calice » des textes centrés autour de la Passion. Les références prennent sens en fonction de la technique de l'exégèse et de sa signification spirituelle.

Cette tension de la digression et du centre se retrouve dans l'opposition, humoristique et polémique à l'égard des exégètes littéralistes, que Claudel établit entre le littéral et le latéral. La latéralité enferme le champ couvert par les figures. Mais elle repose aussi sur une représentation spatiale du texte. Par exemple, dans le commentaire dialogique $\mathrm{Au}$ milieu des vitraux de l'Apocalypse, la Fille métaphorise par la ville chinoise un commentaire auquel se réfère son Père : "C'est comme une rue dans une ville chinoise avec des petites boutiques amusantes de tous les côtés, sans parler des rues latérales qui n'en finissent plus! $\aleph^{40}$ Aussi faut-il constamment recentrer le commentaire divergent. L'intertexte est au cœur de cette tension qui travaille l'écriture. La prolifération de l'hypotexte doit être contrôlée des dispositifs de cadrage du pluriel. Par exemple, nous avons vu que La Rose et le rosaire suit le parcours d'une âme humaine, du bas vers le haut: le modèle de ce parcours est donné par le rosaire dont l'étape ultime « consacre, consomme, parachève cette ascension de l'âme humaine, que typifie la sainte Vierge $»^{41}$. Types, typiques, typifiques, schématiques, « répertoire $»^{42}$ sont des termes qui qualifient la fonction de l'intertexte : l'intertexte par confrontation de deux textes met en évidence le typique, ou confère l'exemplarité, typifie, un objet ou une scène. Si donc il y a digression, elle n'est que le fait d'une lecture littérale : car du pluriel doit se dégager l'essence, ou encore le sens spirituel. L'intertexte comprend et donne une exemplarité qui est elle-même le signe de l'autorité. Claudel résout en fait cette tension dans la citation qu'il fait de la première épître aux Corinthiens : «nous voyons toutes choses comme dans un miroir, en énigme, c'est-à-dire dans une confusion que l'affaire de notre esprit est de débrouiller $»^{43}$. Tandis que la digression maintient la confusion, la fixation dans le type donne « la ressemblance de Dieu ». Le commentaire est ainsi « la transcription extérieure d'un sens intime, l'interprétation de l'idée par le drame moyennant des acteurs appropriés ${ }^{44}$.

Enjeux des pratiques intertextuelles

On peut résumer en ces termes, à ce stade de l'étude, les critères de l'absolu intertextuel claudélien. Il est d'une forte référentialité, reposant sur ce principe que tout texte thématise un pré-texte biblique. Il est d'une intense communicativité, puisque le plus fréquemment, Claudel marque par l'italique, et les références, la 
citation biblique, si bien que l'intention est nettement marquée et univoque de construire une interprétation spirituelle. Cette communicativité et cette intentionnalité se fondent sur une forte sélectivité de l'intertexte, puisque celui-ci intervient comme médiation entre la partie et le tout, l'humain et le divin, la lettre et l'esprit, le matériel et le spirituel : la métonymie commande la démarche claudélienne ${ }^{45}$. L'intertexte est donc structurant, puisqu'il médiatise le sens et assure le passage du littéral au spirituel. Chacun de ces points mérite cependant d'être nuancé. La structuralité et la communicativité nous retiendront.

La structuration par l'intertexte n'est pas formelle, comme elle peut l'être dans de nombreux exemples étudiés par Gérard Genette dans Palimpsestes, ou, par exemple, dans Ulysse de Joyce. La structuralité formelle n'est en général que superficielle dans les commentaires claudéliens et diffère de celle des textes bibliques. En effet, dans $\mathrm{Au}$ milieu des vitraux de l'Apocalypse, le dialogue du Père et de la fille a pour cadre l'observation des vitraux de la Ferté-Millon. Dans Paul Claudel interroge le Cantique des cantiques, Claudel suit verset à verset le poème biblique : la linéarité du texte est constamment rompue par l'intertextualité, et l'unité est à chercher ailleurs qu'en elle. Dans le Livre d'Esther, dont nous avons commenté l'épisode de la remise du sceptre, Claudel ne respecte ni l'ordre de la version hébraïque ni la logique de la version des Septante ${ }^{46}$. Dans Le Livre de Tobie, son choix de textes est sélectif : non seulement le récit de l'aventure de Tobie est inséré dans un récit cadre, celui d'une marche du commentateur accompagnant les deux héros, mais encore Claudel pratique des ellipses et donc opère un choix sélectif :

Et maintenant coupons au court. Et plutôt que de suivre nos deux voyageurs mot à mot et pas à pas, allons les attendre à ce gué qui coche pour nous à travers le Tigre notre chapitre $\mathrm{VI}^{47}$.

Sélection et amplification vont de pair : ainsi, avant cette intervention du locuteur qui ouvre le développement «le poisson", Claudel focalise le commentaire sur les sept prétendants de Sara, Asmodée et les prières simultanées de Sara et Tobie qui font l'objet du chapitre III, 7-17 du texte biblique. Il dramatise son commentaire en faisant des sept prétendants, les "sept candidats évincés à la possession de l'âme humaine ", Sara, les "sept péchés ", " caricature et contre-partie des œuvres de Dieu », les " dons $\mathrm{du}$ Saint-Esprit ${ }^{48}$. On mesure les limites de la structuration formelle dans ce réordonnancement constant $\mathrm{du}$ texte. Conscient du caractère apparemment digressif de son commentaire, quoiqu'il soit concentrique du point de vue de la spiritualité, Claudel recourt fréquemment à la mise en récit ou en drame, ordonnée autour de la catastrophe et de la reconnaissance. Dans Le Livre de Tobie, Claudel écarte les modèles $\mathrm{du}$ récit et du drame, pour leur préférer «la technique du cinéma ${ }^{49}$. C'est que cette formalisation par le drame et le récit est fréquente, au point que Claudel la déplace sur la création ou sur la Bible. La nature, l'histoire, la Bible sont des drames ou des récits. L'ensemble de la création est un drame dont chaque créature est un acteur, comme dans La Rose et le rosaire ${ }^{50}$, et la Bible est aussi un drame :

Un drame architectural, dont l'auteur, le même à travers je ne sais combien de siècles, en même temps que l'action, a inspiré à la fois le théatre, le langage et les acteurs. Il m'était donné enfin de prendre une vue totale, une vue intelligible, et comme a posteriori, de la Terre Promise ${ }^{51}$. directement à la poétique du récit, aristotélicienne, si bien qu'une logique narrative ou 
dramatique recouvre à la surface du texte une logique concentrique, spirituelle. C'est ce double mouvement qui structure Le Livre d'Esther, dont le commentaire est construit en cinq actes.

La communicativité, quant à elle, se fonde sur un intertexte explicite. Dans les premières œuvres, pour des raisons liées à la carrière diplomatique de Claudel et au contexte laïque dans lequel elle se développe, l'hypotexte demeure cryptique. Ni la typographie, ni les références ne permettent d'identifier l'intertexte qui n'a pas statut citationnel: par exemple, la Princesse de Tête d'or qui entre en scène en costume correspond au type de la Princesse fin de siècle, prisonnière du rêve, de la danseuse, dans la lignée des Salomé, même si des vers qu'elle prononce sont des emprunts à l' Ancien testament :

Mais je me tiens au carrefour des chemins, et, dans les villes mêmes, Je me tiens sur les marchés et à la sortie des bals, disant :

"Qui veut changer des mains pleines de mûrons contre des mains pleines d'or?

Et se peser avec son cœur humain un éternel amour ?"52

La Sagesse déclame dans la rue, sur les places elle élève la voix, aux points les plus bruyants, dans la ville, elle appelle, à l'entrée des portes elle prononce ses discours. (Proverbes, I, 20-21; cf. VIII, 2)

Je marche sur la route de la justice

et dans les sentiers de l'équité, procurant à ceux qui m'aiment des ressources et emplissant leurs coffres. (Proverbes, VIII, 20-21)

Structurant le personnage, et en conséquence le sens du drame, l'intertexte n'en reste pas moins peu identifiable au spectateur et au lecteur. Or la structuration et la fonction herméneutique sont soumises à la communicativité. Ce critère est fondamental dans la lecture d'une œuvre telle que celle de Claudel. La question se pose, de manière aussi flagrante, pour Connaissance de l'Est. La critique a salué en ce recueil la poésie de la terre, voire une poésie païenne ${ }^{53}$. Pourtant l'intertexte biblique est inscrit dans le recueil, et signe implicitement la volonté herméneutique de Claudel : face aux objets et paysages orientaux, il s'interroge sur le sens, et pose à son tour la question mallarméenne : «Qu'est-ce que ça veut dire? » Quelques exemples suffiront à mesurer la place, la pertinence, et la fonction de ce décryptage de l'intertexte biblique.

Le poème Le Porc présente à son lecteur, apostrophé au second paragraphe (« Gourmand, paillard!»), la description d'un animal de basse-cour placée au carrefour d'intertextes: Gilbert Gadoffre a montré la référence esthétique à Jules Renard (Histoires d'animaux, Le cochon), sans insister sur les problèmes d'ethos générique que soulève le poème claudélien (registre bas et référence noble à l'Enéide de Virgile), ni sur la signification morale et spirituelle que prend le poème en sa seconde et en sa troisième partie, ni sur les références au genre de la satire et à l'intertexte thomiste ${ }^{54}$. Or cette complexité esthétique, tonale, religieuse s'accroit lorsqu'on rétablit l'intertexte implicite du Nouveau testament, la Deuxième épître de Pierre - la seule que Claudel cite dans ses commentaires, postérieurs - dont le deuxième chapitre, qui stigmatise ceux qui « vont après la chair avec une convoitise immonde » (II, 10), « avec leur nature de bêtes nées pour être prises et détruites » (II, 12), se clôt ainsi : «Il leur est arrivé ce que dit ce proverbe vrai : Le chien retourne à ce qu'il a vomi et la truie lavée se revautre en son bourbier ». (II, $22^{55}$ ) L'intertexte donne la signification réelle 
du poème : expansion paraphrastique de l'intertexte néotestamentaire, Le Porc relève du genre du sermon, où l'apostrophe de l'auditoire précède l'interprétation qu'il faut donner de l'animal au troisième paragraphe. La question posée au commentateur de Connaissance de l'Est est moins celle de la pertinence de cette référence à l'intertexte biblique que des limites de son expansion. Par exemple, le poème La Pluie évoque les pluies abondantes des tropiques : mais l'inondation du paysage qui perd ses limites par une pluie "copieu[se] " peut référer au Psaume LXXII où est ainsi métaphorisé le roi messie qui a du jugement : "Qu'il descende comme la pluie sur le gazon,/ comme des averses inondant la terre !» (6). Ce même verset donne une signification spirituelle à la cinquième séquence du poème Rêves. Le paysage de Connaissance de l'Est, en particulier ses montagnes, ses moissons, les fleuves, le soleil et la lune, sont propres à la poésie des Psaumes: dans le seul Psaume LXXII, se retrouvent tous ces traits. La relation intertextuelle n'est pourtant pas uniquement implicite : elle peut être explicitement textuelle, comme dans le poème Octobre, où la formule «C'en est fait » que mettent en valeur les italiques, réfère directement aux dernières paroles prononcées par le Christ sur la croix : "Consummatum est ». (Jean, XIX, $30^{56}$ ), ou comme dans La dérivation, dont le titre dérive directement du chapitre XXIV de l'Ecclésiastique, où le prophète définit son savoir et son écriture par rapport au fleuve divin, comme le fait Claudel dans son poème qui est à la fois louange et autoréflexion de l'écriture, le « jardin sans fleurs » où pénètre le poète de Connaissance de l'Est (dernier paragraphe) renvoyant aux versets bibliques:

Quant à moi, comme un canal dérivé du fleuve, Comme un cours d'eau je suis sorti vers le paradis.

J'ai dit : « Je vais arroser mon jardin, Je vais saturer mon parterre ». (Ecclésiastique, XXIV, 30-31 ${ }^{57}$ )

La communicativité de l'intertexte relève en fait de la compétence du lecteur et soulève de manière aiguë le problème de la pertinence d'une lecture qui fait l'économie - ou l'impasse - de la référence à la Bible : l'intertextualité confronte ainsi le lecteur au risque de voir sa compréhension d'un texte n'être qu'allégorique, et non historique, s'il en ignore les intertextes. Tout texte est fait des lectures qui l'interprètent $-\mathrm{y}$ compris des lectures pré-textuelles. L'intertextualité est donc nécessaire à une herméneutique complète du texte littéraire.

Intertextualité, auto-réflexivité, retour sur soi

Il est un fait évident: en interrogeant l'intertexte, Claudel opère constamment un retour sur sa propre pratique herméneutique. Définir le Christ comme la page qui permet d'unifier les deux testaments, c'est manifestement trouver dans l'intertexte le fondement de sa propre démarche interprétative. Cette mise en abyme méthodologique se rencontre constamment dans les exégèses claudéliennes. Par exemple, les textes qui régissent les sacrifices hébraïques dans le Deutéronome et le Lévitique deviennent, dans Du sens figuré de l'Ecriture, l'art poétique du commentaire claudélien ${ }^{58}$. Claudel établit une analogie entre la chair et l'âme et la parole qui est union d'esprit et de lettre: l'exégète devient ainsi un sacrificateur, qui, le "scalpel» à la main, cherche «à pénétrer à l'intérieur, à négocier les articulations, à trancher les charnières, là où se fait l'épissure de la chair et de l'esprit " ${ }^{59}$. L' « affreux épisode » du "Lévite d'Ephraïm » est, lui aussi, soumis à ce type d'interprétation sacrificielle dont l'enjeu est l'esprit, le sens unitaire du texte biblique (Livre des Juges, XIX). Un lévite « se voit obligé pendant toute une nuit de livrer [son épouse] au rut bestial des citoyens de Gabaa. Au matin, il la 
retrouve sur le seuil de sa porte, sparsis manibus ». Ce corps violé, que le lévite découpe en "douze morceaux» qu' 'il partage dans un esprit de sommation solennelle aux douze tribus d'Israël», Claudel projette sur lui le texte biblique original, sujet $d^{\prime}$ '« enseignements divers » dont il faut « reconsituter l'unité " ${ }^{60}$. L'exégèse est ainsi une découpe qui est recherche des articulations et du sens unitaire: Claudel divise pour unifier, et non pour parcelliser.

En justifiant par la médiation de l'Ecriture son exégèse, Claudel inscrit sa parole dans la parole de Dieu, ou de l'Esprit Saint. Bien sûr, la dialogicité d'un tel geste est grande, puisque, pour nous en tenir à nos exemples, la lecture de l'épisode du lévite d'Ephraïm oppose l'exégèse unitaire, conforme à celle des Pères de l'Eglise, à l'exégèse historiciste et protestante ${ }^{61}$. Mais l'acte se veut aussi un acte qui maintient constamment le lien avec Dieu : la parole de l'exégète est une parole de foi, où l'âme, désignée par les termes d'anima, de Sagesse, ou encore par la figure de la Vierge Marie, " parl[e] Dieu à Dieu » ${ }^{62}$. La médiation de l'intertexte n'est donc pas uniquement nécessitée par le genre du commentaire : Claudel s'anéantit, en quelque sorte, dans sa "parole d'adoration " ${ }^{63}$. La citation intertextuelle est un acte contemplatif. L'espace et le temps de l'exégèse échappent à toute contingence. Par exemple, Un poète regarde la Croix situe son énonciation dans une église du Nouveau monde où le poète écoute la célébration du Vendredi saint, "hors du temps et pour ainsi dire du lieu ${ }^{64}$. Regarder la croix, c'est écouter les textes bibliques «en notant sur une feuille de papier tout ce qui vient à notre rencontre ${ }^{65}$. Le commentaire est ainsi ce qui vient, et non ce qui se construit. Le moi est pensé comme le dialogue de Dieu avec lui-même qui envahit l'exégète. David figure parfaitement ce moi dont l'œil écoute :

Car, il n'y a pas à se tromper, ces syllabes inouïes, ce que j'ai devant moi, ce que j'entends, ce que je lis, ce que j'épelle, ce que ma fondation ébranlée en ce moment même est à la fois en train de produire et d'écouter : Le Seigneur a dit à mon Seigneur : Prends siège à ma droite! Il y a le Seigneur qui Se parle à Lui-même à travers moi! Il y a le Seigneur qui est en train de Se commander à Lui-même en moi, à un Lui-même en moi conjoint à ma propre substance, exigeant de ma propre personne ${ }^{66}$.

Cette préposition de lieu, en, construit un espace réversible d'inclusions réciproques. En effet, le dialogue de Dieu avec lui-même, par l'intertexte, se fait dans l'âme qui écoute. Mais l'âme, qui "Lui appartient ", "Lui doit existence », " détient de Lui une image qu'elle doit lui restituer », est en Dieu « comme en sa cause permanente ${ }^{67}$. Dieu est en l'âme qui est incluse en Dieu. Cette fusion par la contemplation donne à la présence de l'intertexte un sens religieux très fort. Citer, c'est célébrer et louer Dieu. L'absolu intertextuel fait ainsi du commentaire un poème - Claudel utilise la notion pour désigner ses commentaires - lyrique.

Si l'œil écoute dans la contemplation, cette écoute n'est pas passive. Le dialogue intertextuel exige du moi sa personne : le moi qui est fait, produit, créé, est unique. Commenter, c'est un acte de connaissance qui permet au sujet de co-naître. Toute lecture mise en place par l'intertextualité est unique et singulière, parce ce qu'elle repose, autant qu'elle la révèle, sur l'unicité de l'âme de chaque lecteur. C'est pourquoi Claudel insiste autant sur l'universalité, l'autorité et la solennité de la parole biblique que sur son caractère de "confidence " adressée à chaque lecteur. La Bible s'adresse différemment, pour tous les temps et tous les lieux, à chaque homme. Le verbe causer, utilisé en deux sens, dialoguer et être cause de, désigne cette actualisation de soi dans et par la parole biblique ${ }^{68}$. 

de la biographie à l'intérieur du texte biblique. Cette mutation, qui donne à l'intertexte sa pleine signification sacrificielle, puisque le Christ médiatise les expériences du moi et leur donne sens, «tradui[ $t$ ] en lettres de lumière le texte immonde et contrefait que nous ne nous lassons pas de Lui fournir " ${ }^{69}$. On pourrait reprendre des termes de spritualité, et les appliquer à l'écriture : le commentaire, par l'intertexte qui concentre les faits, a un pouvoir de conversion, ou encore d'édification. Dans L'Epée et le miroir, l'ensemble de l'exégèse est traversé par le souvenir, récurrent, de la conversion de Claudel: à la solennité de la méditation sur les douleurs mariales correspond le parcours du sujet écrivant qui, par les douleurs mariales, purifie et clarifie son âme ${ }^{70}$. Cette parole de Dieu que par les oreilles on lui [=notre converti] a mise dans la bouche, il tâche de la vivre maintenant au ralenti, il écoute le bruit qu'elle fait : tantôt c'est le silence, et tantôt le contact a recommencé : il propose, il pèse, il confère, il compose. Il a fermé la porte et il habite loin des regards ce hiérogramme qui est fait des trois lettres S. O. I. ${ }^{71}$.

L'Epilogue fait le bilan de cette conversion:

L'épée sur moi a fait son œuvre et l'ennemi dont elle m'a débarrassé, la paix qu'elle a établie par la vertu de son tranchant impérial, sont des biens trop précieux pour que je chicane sur les dégâts qu'elle a commis au travers de mes forces physiques ${ }^{72}$.

L'édification du moi, quant à elle, se retrouve dans la métaphore du visage, du vrai visage de soi, révélé par l'écriture exégétique-miroir. Pénétrer l'intériorité du texte biblique par l'intertexte, c'est pénétrer au fond de soi :

Le marteau intérieur est à l'œuvre sur nous, celui du forgeron à tour de bras, et celui obstiné du dentiste, et celui délicat du ciseleur, sans parler de la lime et du papier de verre ${ }^{73}$.

Le poème Préface qui ouvre La Rose et le rosaire fait du dialogue du poète avec la Vierge dans la récitation du rosaire cette édification du visage de soi. Ces vers résument cette composante intime, personnelle, de la pratique intertextuelle de Claudel, que l'on ne saurait réduire ni contenir dans des limites trop étroitement formelles. L'intertexte est un lien, où Dieu dialogue avec lui-même, où le poète dialogue avec Dieu : avec ce lien, Claudel retrouve l'étymologie de religio. L'écriture du commentaire, constamment médiatisée par l'intertexte dont on a souligné la référentialité, la sélectivité, la communicativité, la structuralité, construit un poème et, à travers lui, un sujet de foi. La construction théorique claudélienne n'est insistante autour de la notion d'esprit que pour mieux construire un sujet spirituel. La mosaïque d'intertextes construit ainsi ce que Michel Beaujour appelle un auto-portrait.

Moi, je cause français à la Vierge et elle me répond en latin :

Du moins j'appellerai ainsi, puisque vous le voulez bien, cette espèce de langage à

l'oreille de l'esprit qui n'a rien de sensible et de distinct. (...)

$\mathrm{O}$ Marie, j'ai appris maintenant avec quel respect et quelle attention radieuse et triste,

Vous interrogez au fond de moi cette image de Dieu par quoi j'existe !

Femme, il n'y a langage entre nous que ce cœur qui va violemment à ta rencontre !

Et qui vérifie ce visage en face de lui capable de respirer et de répondre.

Ce visage fait du corps entier et le corps qui n'est fait

Que de la connaissance de Dieu et cette âme qui apparait ! ${ }^{74}$

Cahiers de Narratologie, 13 | 2006 


\section{NOTES}

1. Paul Claudel, Emmaüs, Saül, in O.C., 23, Paris, Gallimard, 1964, p 288. O.C. signifie en note Cuvres complètes. Le chiffre qui suit désigne le tome.

2. O. C. 21, J'aime la Bible, pp. 351-352. Sur ce point, voir aussi O. C. 21, Introduction à l'Apocalypse, pp. 93-95.

3. Euvres en prose, Accompagnements, Mallarmé, Paris, Gallimard, Pléiade, 1965, p. 511.

4. O. C. 21, Le Livre de Job, p. 131. Voir ce qu'il écrit du Livre d'Isaïe in Introduction à Isaïe dans le mot à mot, O.C., 27, pp. 194-195.

5. Du sens figuré de l'écriture, in Introduction au Livre de Ruth, Paris, Desclée de Brouwer, 1938, pp. 101-102. Noté désormais Dsf.

6. O. C. 21, J'aime la Bible, p. 383.

7. Sur l'intention, voir par exemple Etienne Gilson, Le thomisme, Paris, Vrin, 1989,

p. 315-316.

8. Un poète regarde la Croix, Paris, Gallimard, 1935, p. 16-17.

9. Les Aventures de Sophie, Le Livre de Tobie, Paris, Gallimard, 1937, p. 60. Voir Dsf, p. 40. 10. Dsf., p. 28-29. En fait il faut lire Paul Vulliaud, Claudel citant probablement de mémoire. L'ouvrage cité est en fait intitulé La Clef traditionnelle des Evangiles, ce qui se prête moins bien à une réflexion sur les problèmes posés par la traduction de l'hébreu.

11. Les Aventures de Sophie, Le Livre de Tobie, p. 57.

12. Dsf., p. 89. Voir encore O.C. 21, Introduction à l'Apocalypse, p. 96-97, ou J'aime la Bible, p. 352, 354, ou le Livre de Job, p. 99-103.

13. Dsf., p. 90 et p. 91.

14. L'Epée et le miroir, Paris, Gallimard, 1939, p. 74 et p. 192. La citation de la p. 192 est extraite de Jean, 12, 32, dont on ne trouve pas moins de trente occurrences explicites dans l'œuvre exégétique de Claudel, ce qui dit l'importance donnée à ce centrage textuel et spirituel, les deux se condondant, nous l'avons vu (voir Bible de Paul Claudel, Annales littéraires de l'Université de Franche Comté, 690, P. U. F. C., 2000, tome 2, p. 713). Pour Un Poète regarde la Croix, voir p. 67-68 ou p. 156-158. Voir encore O. C. 23, Emmaüs, p. 324-326.

15. Dsf., p. 33.

16. Voir Rimbaud, CEuvres, Une Saison en enfer, Délires I, Vierge folle L'époux infernal, Paris, Garnier, 1960, p. 223.

17. O. C. 23, Seigneur, apprenez-moi à prier, p. 19. Rimbaud écrit : «Quelle vie ! La vraie vie est absente. Nous ne sommes pas au monde ». (op. cit., p. 224)

18. O. C. 21, J'aime la Bible, p. 361.

19. O. C. 21 , J'aime la Bible, p. 354-355. Voir aussi Dsf, p. $35,63,66,69,76-77,79$. On trouve la métaphore du monde-poëme du Créateur p. 61.

20. Je laisse de côté la question de l'analogie. Il n'est pas question dans ce bref développement d'étudier l'ensemble des pratiques intertextuelles, ce qui exigerait un ouvrage.

21. Dsf, p. 69.

22. Voir Un poète regarde la Croix, p. 180. Sur l'intervalle et la suture, pp. 179 et 180 . Sur la Pentecôte, voir Dsf, p. 95.

23. Art poétique, Ed. de Gilbert Gadoffre, Paris, Gallimard, coll. Poésie, 1984, p. 102.

24. Dsf, p. 96. 
25. Dsf, p. 69.

26. O. C. 21, J'aime la Bible, p. 421.

27. O. C. 21, p. 472.

28. Les Aventures de Sophie, p. 49.

29. Claudel reprend ce verset en O.C. 21, J'aime la Bible, p. 435 : "Quel homme, nous dit l'Evangile, réussira à ajouter une coudée à sa taille, fût-ce en s'empoignant par le toupet pour s'extirper de ses bottes ? » (il critique alors la morale de Kant, Plutarque, et l'Art nègre, c'est-à-dire le surréalisme). Voir encore 0.C. 25, Paul Claudel interroge l'Apocalypse, p. 242, et 0. C. 28, p. 338 : «Personne, nous dit l'Evangile, ne peut ajouter une coudée à sa taille. C'est ce que le proverbe américain traduit d'une manière pittoresque, en disant que personne ne peut s'arracher à ses bottes en s'empoignant par le toupet ». 30. Références respectivement à : Exode, 7, 8 ; Esther, 14, et Livre d'Esther, p. 31 ; Genèse, $49,10$.

31. Référence à Thrènes ou Lamentations, 3, 27. « Il est bon pour l'homme de porter le joug dès sa jeunesse ".

32. Voir O. C. 20, L'Epée et le miroir, p. 204, O. C. 22, Paul Claudel interroge le Cantique des Cantiques, p. 122, 162, 196 etc.

33. Théâtre, Paris, Gallimard, Pléiade, 1967, t. 2, p. 1402.

34. Voir notes à cette exégèse in Le Poëte et la Bible, Paris, Gallimard, 1998, t. 1, p. 1530.

35. Voir 0. C. 21, La Rose et le rosaire, Aux lecteurs de ce livre, p. 65-167. Claudel parle de désordre, de morceaux, de liasse ordonnée par un parcours, de "cette âme misérable » au « visage d'en haut » ou par un « lien, une échelle qu'il nous est permis de gravir marche à marche et grain à grain et qui est appelée le Rosaire ».

36. Elle peut apparaître - même si, en l'occurrence elle ne l'est pas. Je reviens sur la compréhension réelle de cela lorsque j'évoque la fonction autoréflexive de l'intertextualité.

37. Un poète regarde la Croix, p. 11-15.

38. Un poète regarde la Croix, p. 7.

39. Genèse, XLIV, 2, 5.

40. Le Poëte et la Bible, p. 195.

41. O. C. 21, La Rose et le rosaire, Aux lecteurs de ce livre, p. 165.

42. Sur le répertoire, voir Introduction à l'Apocapypse, O. C. 21, p. 97 . Les pages 96-98 développent une réflexion sur le type.

43. Dsf, p. 74.

44. Dsf, p. 75. Voir p. 62-63: «Rien ne se passe que par rapport à ce qui ne passe pas. Nous voyons tout comme dans un miroir, nous dit l'apôtre. Mais ce miroir, qui n'est autre que l'éternité divine, du fait même de sa pureté impénétrable, a, comme la plaque photographique, une vertu préhensible d'arrêt et de fixation. Elle retient. Elle est comme l'écriture, qui se saisit de la parole et l'empêche de bouger, qui la transforme en un document et en un mot, non seulement signficatif de lui-même, mais clarificatif de la phrase et de l'ensemble dont il est une partie ».

45. Je me réfère aux critères énoncés par M. Pfister, Konzepte des Intertextualität, Zur Systemreferenz, Intertextualität (Formen, Funktionen, anglistische Fallstudien), Ed. U. Broich et M. Pfister, Tübingen, 1985, pp. 1-30 et pp. 52-58.

46. Sur ce point, voir les commentaires de Dominique Millet-Gérard, in Le Poëte et la Bible, p. 1520.

47. Le Livre de Tobie, in Les Aventures de Sophie, p. 92.

48. Le Livre de Tobie, in Les Aventures de Sophie, pp. 64, 65, 66. 
49. Le Livre de Tobie, in Les Aventures de Sophie, p. 56. Mais dans son mimodrame L'Histoire de Tobie et de Sara, il recourt au drame et à l'écran de cinéma dans la mise en scène. Sur la question du récit voir notre "Catastrophe et révélation dans la lecture de l'Apocalypse de Jean de Paul Claudel et Jean Grosjean", actes du colloque L'Apocalypse organisé par le Centre Jacques Petit, janvier 1999 (P. U. F. C., 2001).

50. O. C. 21, p. 294-295.

51. O. C. 21, J'aime la Bible, p. 352.

52. Théâtre, Paris, Gallimard, Pléiade, 1967, t. 1. p. 65.

53. Sur ce point, voir Pascale Alexandre, « Du "visiteur insolite" à "P’tit Paul" : jalons pour une réception de l'œuvre claudélienne ", Revue d'Histoire littéraire de la France, $2000, \mathrm{n}^{\circ} 2$, p. 287-304.

54. Sur ce point, voir Connaissance de l'Est, édition critique établie par Gilbert Gadoffre, Paris, Mercure de France, 1973, p. 17 sv. Voir Claude-Pierre Pérez, Le défini et l'inépuisable, Annales littéraires de l'université de Besançon, Les Belles Lettres, 1995, p. 74 sv. Sur l'intertextualité thomiste, voir notre Genèse de la poétique de Paul Claudel, « comme le grain hors du furieux blutoir », Paris, Champion, coll. Littérature de notre temps, 2001.

55. Le chien apparaît dans le poème de Claudel. Le premier proverbe renvoie à Proverbes, XXVI, 11. Dans O. C. 20, Claudel traduit : "C'est (...) la truie qui se tourne et se retourne voluptueusement dans son bourbier (Sus lota in volutabiro luti) (p. 114). Voir aussi O. C. 25, Paul Claudel interroge l'Apocalypse, p. 367 : «Sus in volutabro! La jouissance présente, immédiate, et allez donc ! »Claudel écrit dans Le Porc : «C'est une jouissance profonde, solitaire, consciente, intégrale ». Eeuvre poétique, Paris, Gallimard, Pléiade, 1967 , p. 58)

56. Formule traduite par « C'est fini », mais que Claudel traduit par « Tout est consommé »(O. C. 19, p. 431) ou par «C'est fait» (O. C. 20, p. 81).

57. Aussi le fleuve est-il métaphore de la Sagesse (Ecclésiastique, XXIV, 27). On retrouve à la fin de la première des Cinq Grandes Odes, Les Muses, la métaphore de l'irrigation dérivée, elle aussi, de l'Ecclésiastique (XXIV, 30-34). On ne peut mieux dire que l'écriture dérive de l'intertexte biblique. Nous avons là encore un exemple de la fonction autoréflexive de l'intertexte.

58. Voir notre « Disjecti membra poetae : le tout et la partie dans l'exégèse claudélienne, à paraître in Actes du colloque L'Ecriture de l'exégèse dans l'œuvre de Paul Claudel, 8-10 mars 2001, Université de Toulouse- Le Mirail.

59. Dsf, pp. 40-41. Pour l'ensemble du texte, voir pp. 39-42.

60. O. C. 21, J'aime la Bible, p. 384-385. Voir O. C. 20, pp. 326-329, O. C. 23, Emmaüs, p. 276-277, p. 309.

61. O. C. 21, J'aime la Bible, p. 384.

62. O. C. 21, La Rose et le rosaire, p. 224.

63. Art poétique, p. 61.

64. Op. cit., p. 7.

65. Op. cit., p. 9.

66. O. C. 23, Emmaüs, p. 325.

67. Sur la présence de Dieu, in Présence et prophétie, Egloff, Fribourg, L. U. F., Paris, 1942, p. 15.

68. Dsf, p. 31, voir p. 101-102.

69. Voir L'Epée et le miroir, p. 85.

70. Voir L'Epée et le miroir, p. 19, 21, 26, 33, 68, 78, 80, 88, 95 etc. 
71. L'Epée et le miroir, p. 43.

72. L'Epée et le miroir, p. 205.

73. L'Epée et le miroir, p. 177, cf. pp. 27, 38.

74. O. C. 21, La Rose et le rosaire, p. 163-164.

\section{RÉSUMÉS}

Pourquoi qualifier d'absolue l'intertextualité que Claudel pratique dans ses exégèses bibliques? Il faut comprendre l'épithète dans son sens scripturaire, tout texte biblique convoquant par association d'autres textes biliques : l'intertexte est ainsi structurant, communicatif, référentiel, puisqu'il permet d'intégrer la partie dans une totalité, d'établir le lien entre la parole humaine du commentateur et l'Esprit saint, et de référer tout élément à un co-texte qui le transcende. Le préfixe inter, dans cette écriture, prend une valeur forte : l'interprète établit des connexions qui suppriment les intervalles. Il faut relativiser néanmoins ces fonctions : la structuralité est aussi le fait de la digression ou de la mise en récit, et la communicativité s'avère plus problématique quand l'intertexte est implicite.

L'épithète prend un sens plus métaphysique lorsque l'intertextualité est étudiée en termes d'autorité, d'inscription de la parole humaine dans le verbe divin, et de fusion du sujet écrivant dans la contemplation. L'intertextualité se fait ainsi parole de confidence et de prière, où le fait biographique prend sens dans l'absolu divin inscrit dans le texte biblique. Commentaire et découverte de soi sont donc indissociables.

\section{AUTEUR}

\section{DIDIER ALEXANDRE}

Université de Toulouse-Le Mirail 